\title{
$\beta$-functions in higher dimensional field theories
}

\author{
J.A. Gracey \\ Theoretical Physics Division, Department of Mathematical Sciences, University of Liverpool, \\ P.O. Box 147, Liverpool, L69 3BX, United Kingdom \\ E-mail: gracey@liverpool.ac.uk
}

We review recent activity in the construction of the renormalization group functions for $O(N)$ scalar and gauge theories in six and higher dimensions. The theories lie in their respective universality classes at the Wilson-Fisher fixed point. The critical exponents at this fixed point in the various dimensions are all in agreement with the known exponents determined in the large $N$ expansion.

Loops and Legs in Quantum Field Theory

24-29 April 2016

Leipzig, Germany

*Speaker. 


\section{Introduction}

Dimensional regularization is the canonical method of controlling the divergences when renormalizing a quantum field theory in perturbation theory. The theory is extended from the critical dimension $D$ to $d$ where $d=D-2 \varepsilon$ and $\varepsilon$ is the regularizing parameter. The poles in $\varepsilon$ are then absorbed in renormalization constants prior to the construction of the renormalization group functions. The process is complete when these functions are determined in $d=D$ dimensions. What is less apparent in the procedure is the connectivity different theories, with the same underlying symmetry, have with each other in different dimensions. This connection derives from the critical point renormalization group equation and the Wilson-Fisher (WF) fixed point which is defined as the non-trivial zero of the $\beta$-function in $d$-dimensions. This connectivity has seen a recent revival in analysing conformal field theories beyond two dimensions as well as related interest in the $a$ theorem, ultraviolet-infrared duality across dimensions and conformal windows. Here we review the renormalization group functions of scalar and gauge theories in dimensions higher than four in their respective universality classes.

\section{Scalar theories}

We begin by reprising the picture of a tower of theories in $d$-dimensions all lieing in the same universality class at the WF fixed point by considering scalar theories with an $O(N)$ symmetry. The base theory is in two dimensions which is the $O(N)$ nonlinear $\sigma$ model with the Lagrangian

$$
L^{\sigma}=\frac{1}{2}\left(\partial_{\mu} \phi^{i}\right)^{2}+\frac{1}{2} \sigma\left(\phi^{i} \phi^{i}-\frac{1}{\lambda}\right)
$$

where $\lambda$ is the coupling constant and $\sigma$ is a Lagrange multiplier field. Throughout the set of theories in the same universality class the two fields have dimensions $\left[\phi^{i}\right]=d / 2-1$ and $[\sigma]=2$ in $d$-dimensions. While (2.1) is non-renormalizable perturbatively it is renormalizable in the $1 / N$ expansion which is a dimensionless coupling parameter in $d$-dimensions. This point of view of the renormalizability is the key to understanding the dimensional connectivity. The common feature of theories in the same universality class is the core interaction such as that in (2.1). For instance, relative to four dimensions (2.1) is critically equivalent to $O(N) \phi^{4}$ theory which can be formulated in two ways since

$$
L^{(4)}=\frac{1}{2}\left(\partial_{\mu} \phi^{i}\right)^{2}-\frac{g}{8}\left(\phi^{i} \phi^{i}\right)^{2}=\frac{1}{2}\left(\partial_{\mu} \phi^{i}\right)^{2}+\frac{1}{2} \sigma \phi^{i} \phi^{i}-\frac{\sigma^{2}}{2 g} .
$$

In the latter Lagrangian $\sigma$ is an auxiliary field and appears quadratically rather than linearly to ensure perturbative renormalizability in the critical dimension. These additional $\sigma$ dependent parts of a Lagrangian over and above the core interaction are termed spectators since they are only present in specific dimensions. Once (2.1) and (2.2) are viewed in this way the method to construct the Lagrangians in the universal tower is evident and requires a core interaction and renormalizability.

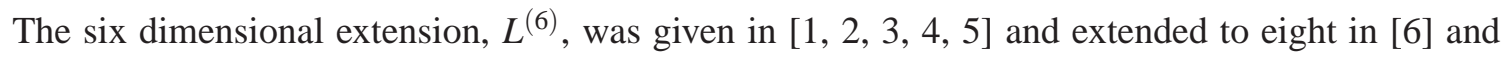
their respective Lagrangians are

$$
L^{(6)}=\frac{1}{2}\left(\partial_{\mu} \phi^{i}\right)^{2}+\frac{1}{2}\left(\partial_{\mu} \sigma\right)^{2}+\frac{g_{1}}{2} \sigma \phi^{i} \phi^{i}+\frac{g_{2}}{6} \sigma^{3}
$$




$$
L^{(8)}=\frac{1}{2}\left(\partial_{\mu} \phi^{i}\right)^{2}+\frac{1}{2}(\square \sigma)^{2}+\frac{1}{2} g_{1} \sigma \phi^{i} \phi^{i}+\frac{1}{6} g_{2} \sigma^{2} \square \sigma+\frac{1}{24} g_{3}^{2} \sigma^{4}
$$

where the number of independent couplings increases with dimension. For all of these theories the critical exponents which are derived from the renormalization group functions have been computed in the large $N$ expansion, [0, 8, 9, 10]. For instance, the first three terms of the exponents

$$
\eta=\gamma_{\phi}\left(g_{c}\right), \omega=\beta^{\prime}\left(g_{c}\right)
$$

are known, [7, 8, 9, 10], as functions of $d$ where $\gamma_{\phi}(g)$ is the wave function anomalous dimension and $g_{c}$ represents the vector of critical couplings. It is worth noting that the derivation of large $N$ exponents uses analytic regularization of the Feynman integrals and not dimensional regularization which is effectively the reason why one can study field theories in $d$-dimensions. Moreover, the large $N$ method is applicable to non-abelian gauge theories but $N_{f}$, the number of quark flavours, is used as the expansion parameter rather than the number of colours.

Recent activity for $L^{(6)}$ has involved the extension of the three loop results of [11, 12] to the $O(N)$ case, [3, 5], and then to four loops, [13]. For the latter computation one can derive all the basic renormalization group functions from evaluating a 2-point function. This is because in six dimensions a $1 /\left(k^{2}\right)^{2}$ propagator is infrared safe unlike in four dimensions. Therefore to extract the coupling constant renormalization one can nullify one external leg momentum on the vertex function relegating it effectively to a 2-point function evaluation. Such a nullification can be accommodated within the usual renormalization of the field 2-point function, [13]. This approach substantially reduces the number of Feynman graphs to be evaluated. However, to achieve this we have used the Laporta algorithm, [14], and specifically the REDUZE encoding of it, [15, 16], to construct all the integration by parts relations between the required integrals. The final step requires the substitution of the basic master integrals. As the 2-point four loop master integrals are known in four dimensions, [17], we can determine the corresponding ones in six dimensions by applying Tarasov's method, [18, 19]. We use FORM, [20], throughout to handle the underlying algebra. The main results are provided in [13] but we note that the renormalization group functions for the $O(1)$ version of $L^{(6)}$ are, [11, 12, 13],

$$
\begin{aligned}
\beta(g)= & \frac{3}{8} g^{3}-\frac{125}{288} g^{5}+5\left[2592 \zeta_{3}+6617\right] \frac{g^{7}}{41472} \\
& +\left[-4225824 \zeta_{3}+349920 \zeta_{4}+1244160 \zeta_{5}-3404365\right] \frac{g^{9}}{1492992} \\
\gamma_{\phi}(g)= & -\frac{1}{12} g^{2}+\frac{13}{432} g^{4}+\left[2592 \zeta_{3}-5195\right] \frac{g^{6}}{62208} \\
& +\left[10080 \zeta_{3}+18144 \zeta_{4}-69120 \zeta_{5}+53449\right] \frac{g^{8}}{248832}
\end{aligned}
$$

where $\zeta_{n}$ is the Riemann zeta-function. Finally, all the renormalization group functions evaluated for the $O(N)$ versions of (2.3), [2, 3, 6, 13], are in full agreement with the known large $N$ exponents which supports the Wilson's vision of a tower of theories in $d$-dimensions within a universal underlying theory. 


\section{Gauge theories}

We can also extend the process to non-supersymmetric gauge theories. First, we focus on six-dimensional QCD. The gauge fixed Lagrangian in a linear covariant gauge is, [6],

$$
\begin{aligned}
L_{\mathrm{GI}}^{(6)}= & -\frac{1}{4}\left(D_{\mu} G_{v \sigma}^{a}\right)\left(D^{\mu} G^{a v \sigma}\right)+\frac{g_{2}}{6} f^{a b c} G_{\mu \nu}^{a} G^{b \mu \sigma} G_{\sigma}^{c \nu}+i \bar{\psi}^{i I} \not D \psi^{i I} \\
& -\frac{1}{2 \alpha}\left(\partial_{\mu} \partial^{v} A_{v}^{a}\right)\left(\partial^{\mu} \partial^{\sigma} A_{\sigma}^{a}\right)-\bar{c}^{a} \square\left(\partial^{\mu} D_{\mu} c\right)^{a}
\end{aligned}
$$

where $\alpha$ is the gauge parameter. In six dimensions there are two independent gluonic operators, [21], as a consequence of the Bianchi identities and we have chosen to use a 2-leg and a 3-leg operator. The latter is important in effective field theories in four dimensions. Though in six dimensions a 4-fermi operator has dimension 10 and hence is absent in (3.1). As a consequence the gluon and ghost propagators have double poles in the squared momentum unlike in four dimensions. Structurally (3.1) is similar to the eight dimensional $O(N)$ scalar theory, [6]. However, as an aside the propagators of (3.1) would lead to a confining inter-quark potential in four dimensions. Indeed there is a Schwinger-Dyson solution of four dimensional QCD which constructs the effective infrared Lagrangian, [22], and takes the precise form (3.1) with $g_{2}$ massive. Continuing this theme (3.1) can be extended to include lower dimension operators with

$$
\begin{aligned}
L_{m}^{(6)}= & L^{(6)}+m_{1} \bar{\psi}^{i I} \psi^{i I}-\frac{1}{4} m_{2}^{2} G_{\mu \nu}^{a} G^{a \mu \nu}-\frac{1}{2 \alpha} m_{3}^{2}\left(\partial^{\mu} A_{\mu}^{a}\right)^{2} \\
& -m_{3}^{2} \bar{c}^{a}\left(\partial^{\mu} D_{\mu} c\right)^{a}-\frac{1}{2} m_{4}^{4} A_{\mu}^{a} A^{a \mu}+m_{4}^{4} \alpha \bar{c}^{a} c^{a} .
\end{aligned}
$$

Here $m_{i}$ are masses to ensure each term is dimension six. Interestingly the Landau gauge propagators derived from (3.2) are formally the same as those which are used to model the infrared structure of the propagators on the lattice in four dimensions, [23]. For instance,

$$
\begin{aligned}
\left.\left\langle A_{\mu}^{a}(p) A_{v}^{b}(-p)\right\rangle\right|_{\alpha=0} & =-\frac{\delta^{a b}}{\left[\left(p^{2}\right)^{2}+m_{2}^{2} p^{2}+m_{4}^{4}\right]}\left[\eta_{\mu \nu}-\frac{p_{\mu} p_{v}}{p^{2}}\right] \\
\left.\left\langle c^{a}(p) \bar{c}^{b}(-p)\right\rangle\right|_{\alpha=0} & =-\frac{\delta^{a b}}{p^{2}\left[p^{2}+m_{3}^{2}\right]} .
\end{aligned}
$$

Returning to the massless Lagrangian we have calculated the wave function anomalous dimensions and the $\beta$-functions to two loops for non-zero $\alpha$. For the latter we evaluated the three 3 -point vertices at the fully symmetric point with non-zero momenta flowing through each external leg. Unlike in a renormalization of four dimensional QCD nullifying an external leg momentum is not infrared safe in six dimensions as such an operation would produce propagators of the form $1 /\left(k^{2}\right)^{4}$ where $k$ is a loop momentum. By renormalizing these three vertices and obtaining the same $\overline{\mathrm{MS}}$ results for the renormalization of $g_{1}$ is a non-trivial check on our computation. Like the scalar theory analysis we have again used the Laporta algorithm, [14], to obtain our renormalization group functions. For instance, the two $\overline{\mathrm{MS}} \beta$-functions are, [6],

$$
\begin{aligned}
\beta_{1}\left(g_{1}, g_{2}\right)= & {\left[-249 C_{A}-16 N_{f} T_{F}\right] \frac{g_{1}^{3}}{120} } \\
& +\left[-50682 C_{A}^{2} g_{1}^{3}+2439 C_{A}^{2} g_{1}^{2} g_{2}+3129 C_{A}^{2} g_{1} g_{2}^{2}-315 C_{A}^{2} g_{2}^{3}-1328 C_{A} N_{f} T_{F} g_{1}^{3}\right.
\end{aligned}
$$




$$
\begin{gathered}
\left.-624 C_{A} N_{f} T_{F} g_{1}^{2} g_{2}+96 C_{A} N_{f} T_{F} g_{1} g_{2}^{2}-3040 C_{F} N_{f} T_{F} g_{1}^{3}\right] \frac{g_{1}^{2}}{4320} \\
\beta_{2}\left(g_{1}, g_{2}\right)=\left[81 C_{A} g_{1}^{3}-552 C_{A} g_{1}^{2} g_{2}+135 C_{A} g_{1} g_{2}^{2}-15 C_{A} g_{2}^{3}+104 N_{f} T_{F} g_{1}^{3}-48 N_{f} T_{F} g_{1}^{2} g_{2}\right] \frac{1}{120}
\end{gathered}
$$

where for space reasons we only note the one loop term of $\beta_{2}\left(g_{1}, g_{2}\right)$. The full expression is given in [6]. We note that the gauge coupling constant is asymptotically free for all (positive) values of $N_{f}$. A final check on the calculation of the renormalization group functions is if the critical exponents derived from them at the WF fixed point agree with the large $N_{f}$ exponents computed using Vasiliev's method. In this instance the base theory in two dimensions which serves to seed this fixed point universal theory is the non-abelian Thirring model (NATM) which can be written in two ways as

$$
L^{\mathrm{NATM}}=i \bar{\psi}^{i} \not \partial \psi^{i}+\frac{\tilde{g}}{2}\left(\bar{\psi}^{i} T^{a} \gamma^{\mu} \psi^{i}\right)^{2}=i \bar{\psi}^{i} \not D \psi^{i}-\frac{1}{2} A_{\mu}^{a} A^{a \mu}
$$

where in strictly two dimensions $A_{\mu}^{a} \propto \bar{\psi}^{i} T^{a} \gamma_{\mu} \psi^{i}$ is an auxiliary field. Various large $N_{f}$ critical exponents are known, [24, 25, 26], and expanding these in an $\varepsilon$-expansion relative to six dimensions the two loop renormalization group functions are in total agreement. That this happens for (3.1) indicates that it is correct which is not as remarkable as the observation of [27] that (3.5) does not contain gluonic vertices. The large $N_{f} d$-dimensional critical exponents derived from (3.5) contain information on the contribution from the triple, quartic and quintic vertices of the higher dimensional gauge theories.

Evidence for the connection of the field theories across the dimensions is not just restricted to the basic renormalization group functions. We have also computed the two loop $\overline{\mathrm{MS}}$ corrections to the anomalous dimensions, $\gamma_{(n)}\left(g_{1}, g_{2}\right)$, of the flavour non-singlet twist-2 Wilson operators $\bar{\psi} \gamma^{\mu_{1}} D^{\mu_{2}} \ldots D^{\mu_{n}} \psi$ using 3.1. For the lowest moments we find

$$
\begin{aligned}
\gamma_{(2)}\left(g_{1}, g_{2}\right)= & 2 C_{F} g_{1}^{2} \\
& +\left[26841 C_{A} g_{1}^{2}-1200 C_{A} g_{1} g_{2}-600 C_{A} g_{2}^{2}-4200 C_{F} g_{1}^{2}+1264 g_{1}^{2} N_{f} T_{F}\right] \frac{C_{F} g_{1}^{2}}{1800} \\
\gamma_{(3)}\left(g_{1}, g_{2}\right)= & \frac{49}{15} C_{F} g_{1}^{2} \\
& +\left[186321 C_{A} g_{1}^{2}-10950 C_{A} g_{1} g_{2}-4200 C_{A} g_{2}^{2}-23564 C_{F} g_{1}^{2}+9104 g_{1}^{2} N_{f} T_{F}\right] \frac{7 C_{F} g_{1}^{2}}{54000} .
\end{aligned}
$$

Again evaluating these at the WF fixed point to determine the critical exponent they are in exact agreement with the leading order large $N_{f}$ exponent of [28].

One application of (3.4) is that the location of the conformal window can be determined in an $\varepsilon$ expansion with $d=6-2 \varepsilon$. In four dimensional $S U(3)$ QCD the window is at $9 \leq N_{f} \leq 16$, [29]. By numerically solving

$$
\beta_{1}\left(g_{1}, g_{2}\right)=\beta_{2}\left(g_{1}, g_{2}\right)=0, \frac{\partial \beta_{1}}{\partial g_{1}} \frac{\partial \beta_{2}}{\partial g_{2}}-\frac{\partial \beta_{1}}{\partial g_{2}} \frac{\partial \beta_{2}}{\partial g_{1}}=0
$$


we find one real solution labelled by $A$ which is, [6],

$$
N_{f(A))}=2.797566 \frac{C_{A}}{T_{F}}+\left[2.198165 C_{F}-3.432003 C_{A}\right] \frac{\varepsilon}{T_{F}}+O\left(\varepsilon^{2}\right)
$$

where we retain the four dimensional trace convention. For $S U(3)$ we have

$$
\left.N_{f(A)}\right|_{S U(3)}=16.785398-14.730246 \varepsilon+O\left(\varepsilon^{2}\right) .
$$

Unlike the situation with $O(N)$ scalar theories the boundary in strictly six dimensions is the same as in four dimensions.

Further insight into the structure of higher dimensional gauge theories can be gained by focusing on the extension of Quantum Electrodynamics (QED) to six and eight dimensions. This is because one can compute to higher order than in the non-abelian case especially in eight dimensions where there are a substantial number of 4-gluon operators. The respective Lagrangians are, [30, 6],

$$
\begin{aligned}
\left.L^{(6)}\right|_{U(1)}= & -\frac{1}{4}\left(\partial_{\mu} F_{v \sigma}\right)\left(\partial^{\mu} F^{v \sigma}\right)-\frac{1}{2 \alpha}\left(\partial_{\mu} \partial^{v} A_{v}\right)\left(\partial^{\mu} \partial^{\sigma} A_{\sigma}\right)+i \bar{\psi}^{i} \not D \psi^{i} \\
\left.L^{(8)}\right|_{U(1)}= & -\frac{1}{4}\left(\partial_{\mu} \partial_{v} F_{\sigma \rho}\right)\left(\partial^{\mu} \partial^{v} F^{\sigma \rho}\right)-\frac{1}{2 \alpha}\left(\partial_{\mu} \partial^{v} A_{v}\right)\left(\partial^{\mu} \partial^{\sigma} A_{\sigma}\right) \\
& +i \bar{\psi}^{i} \not D \psi^{i}+\frac{g_{2}^{2}}{32} F_{\mu \nu} F^{\mu v} F_{\sigma \rho} F^{\sigma \rho}+\frac{g_{3}^{2}}{8} F_{\mu v} F^{\mu \sigma} F_{v \rho} F^{\sigma \rho}
\end{aligned}
$$

where we have extended the gauge fixing sector to eight dimensions for a linear covariant gauge. The renormalization group functions of each theory are known to various loop orders, [30, 6], including the electron mass anomalous dimensions. In all cases the critical exponents derived from these are in full agreement with the corresponding critical exponents to whatever order they are known in large $N_{f},[31,24,26]$. This again substantiates the picture of a tower of theories connected via the WF fixed point. Several general features emerge in the QED analysis. First, we have verified the result of [30] that six dimensional QED is asymptotically free. By contrast the eight dimensional theory is like its four dimensional partner. Under the assumption that there are no triple photon vertices in any QED formulation in higher dimensions then $D$-dimensional QED is asymptotically free in $D=2+4 r$ dimensions for integer $r \geq 1$. For both Lagrangians (3.10) we have explicitly checked that the Ward-Takahashi identity holds. In addition a novel feature emerges for the electron anomalous dimension in a linear covariant gauge. In four dimensions the gauge parameter appears only in the one loop term, [32, 33], and is absent thereafter in any explicit evaluation. The six and eight dimensional theories share the same property to three and two loops respectively. Therefore, if a rigorous proof of this observation emerges it should be applicable to all dimensions.

\section{Discussion}

We have reviewed recent work in six and higher dimensional scalar and gauge field theories and provided solid evidence for the picture of a tower of connected theories in $d$-dimensions accessed by the WF fixed point. This extends from strictly two dimensions where conformal field 
theories give the foundation for the connection. Results in gauge theories show a similar vision but with the observation that structurally eight dimensional scalar theory is similar to six dimensional QCD. From the higher dimensional quantum field theory side the next stage is to extend loop computations to higher order to refine the fixed point structure as will as to gain more insight into the operators which drive any infrared fixed points in QCD in the context of the underlying universal theory. Form the computational point of view one question is whether there is a deeper connection of the Tarasov construction of relating $d$ - and $(d+2)$-dimensional Feynman integrals with the underlying field theories. In other words is there a way of proceeding more fundamentally via a path integral construction without having to make the connection at the renormalization group function level?

\section{Acknowledgements}

This work was carried out with the support of the STFC Consolidated Grant ST/L000431/1.

\section{References}

[1] K. Yonekura, Perturbative c-theorem in d-dimensions, JHEP 1304 (2013) 011 [arXiv:1212.3028].

[2] L. Fei, S. Giombi \& I.R. Klebanov, Critical $O(N)$ models in $6-\varepsilon$ dimensions, Phys. Rev. D90 (2014) 025018 [arXiv: 1404.1094$]$.

[3] L. Fei, S. Giombi \& I.R. Klebanov, Three loop analysis of the critical $O(N)$ models in $6-\varepsilon$ dimensions, Phys. Rev. D91 (2015) 045011 [arXiv: 1411.1099 ].

[4] B. Grinstein, D. Stone, A. Stergiou \& M. Zhong, Challenge to the a theorem in six dimensions, Phys. Rev. Lett. 113 (2014) 231602 [arXiv: 1406.3626$].$

[5] L. Fei, S. Giombi, I.R. Klebanov \& G. Tarnopolsky, Critical Sp $(N)$ models in $6-\varepsilon$ dimensions and higher spin dS/CFT, JHEP 1509 (2015) 076 [arXiv: 1502 . 07271].

[6] J.A. Gracey, Six dimensional QCD at two loops, Phys. Rev. D93 (2015) 025025 [arXiv:1512.04443].

[7] A.N. Vasil'ev, Yu.M. Pismak \& J.R. Honkonen, Simple method of calculating the critical indices in the 1/N expansion, Theor. Math. Phys. 46 (1981) 104.

[8] A.N. Vasil'ev, Yu.M. Pismak \& J.R. Honkonen, $1 / N$ expansion: calculation of the exponents $\eta$ and $v$ in the order $1 / N^{2}$ for arbitrary number of dimensions, Theor. Math. Phys. 47 (1981) 465.

[9] A.N. Vasil'ev, Yu.M. Pismak \& J.R. Honkonen, $1 / n$ expansion: calculation of the exponent $\eta$ in the order $1 / n^{3}$ by the conformal bootstrap method, Theor. Math. Phys. 50 (1982) 127.

[10] D.J. Broadhurst, J.A. Gracey \& D. Kreimer, Beyond the triangle and uniqueness relations: Nonzeta counterterms at large N from positive knots, Z. Phys. C75 (1997) 559 [hep-th/9607174].

[11] O.F. de Alcantara Bonfim, J.E. Kirkham \& A.J. McKane, Critical exponents to order $\varepsilon^{3}$ for $\phi^{3}$ models of critical phenomena in $6-\varepsilon$ dimensions, J. Phys. A13 (1980) L247.

[12] O.F. de Alcantara Bonfim, J.E. Kirkham \& A.J. McKane, Critical exponents for the percolation problem and the Yang-Lee edge singularity, J. Phys. A14 (1981) 2391. 
[13] J.A. Gracey, Four loop renormalization of $\phi^{3}$ theory in six dimensions, Phys. Rev. D92 (2015) 025012 [arXiv:1506.03357].

[14] S. Laporta, High precision calculation of multiloop Feynman integrals by difference equations, Int. J. Mod. Phys. A15 (2000) 5087 [hep-ph / 0207004 ].

[15] C. Studerus, Reduze - Feynman integral reduction in C++, Comput. Phys. Commun. 181 (2010) 1293 [arXiv:0912.2546].

[16] A. von Manteuffel \& C. Studerus, Reduze 2 - distributed Feynman integral reduction, arXiv:1201.4330 [arXiv:1201.4330].

[17] P.A. Baikov, K.G. Chetyrkin \& J.H. Kühn, Four loop massless propagators: an algebraic evaluation of all master integrals, Nucl. Phys. B837 (2010) 186 [arXiv: 1004 .1153].

[18] O.V. Tarasov, Connection between Feynman integrals having different values of the space-time dimension, Phys. Rev. D54 (1996) 6479 [hep-ph/9606018].

[19] O.V. Tarasov, Generalized recurrence relations for two loop propagator integrals with arbitrary masses, Nucl. Phys. B502 (1997) 455 [hep-ph/ 9703319$].$

[20] J.A.M. Vermaseren, New features of FORM, math-ph/0010025.

[21] D.I. Kazakov, Ultraviolet fixed points in gauge and SUSY field theories in extra dimensions, JHEP 0303 (2002) 020 [hep-th/ 0209100 ].

[22] A.I. Alekseev, B.A. Arbuzov \& V.A. Baikov, Infrared asymptotics of gluon Green's functions in Quantum Chromodynamics, Theor. Math. Phys. 52 (1982) 739.

[23] A. Cucchieri, D. Dudal, T. Mendes \& N. Vandersickel, Modeling the gluon propagator in Landau gauge: lattice estimates of pole masses and dimension-two condensates, Phys. Rev. D85 (2012) 094513 [arXiv: 1111.2327].

[24] J.A. Gracey, Quark, gluon and ghost anomalous dimensions at $O\left(1 / N_{f}\right)$ in Quantum Chromodynamics, Phys. Lett. B318 (1993) 177 [hep-th/9310063].

[25] J.A. Gracey, The $Q C D \beta$-function at $O\left(1 / N_{f}\right)$, Phys. Lett. B373 (1996) 178 hep-ph / 9602214 ].

[26] M. Ciuchini, S.É. Derkachov, J.A. Gracey \& A.N. Manashov, Computation of quark mass anomalous dimension at $O\left(1 / N_{f}^{2}\right)$ in Quantum Chromodynamics, Nucl. Phys. B579 (2000) 56 [hep-ph/9912221].

[27] A. Hasenfratz \& P. Hasenfratz, The equivalence of the $S U(N)$ Yang-Mills theory with a purely fermionic model, Phys. Lett. B297 (1992) 166 [hep-lat/9207017].

[28] J.A. Gracey, Anomalous dimension of nonsinglet Wilson operators at $O\left(1 / N_{f}\right)$ in deep inelastic scattering, Phys. Lett. B322 (1994) 141 [hep-ph/ 9401214$].$

[29] T. Banks \& A. Zaks, On the phase structure of vector-like gauge theories with massless fermions, Nucl. Phys. B196 (1982) 189.

[30] S. Giombi, I.R. Klebanov \& G. Tarnopolsky, Conformal QED, , F-theorem and the $\varepsilon$ expansion, J. Phys. A49 (2016) 135403 [arXiv: 1508 . 06354].

[31] A. Palanques-Mestre \& P. Pascual, The $N_{f}$ expansion of the $\gamma$ and $\beta$ functions in $Q E D$, Commun. Math. Phys. 95 (1984) 277.

[32] K. Johnson \& B. Zumino, Gauge dependence of the wave-function renormalization constant in Quantum Electrodynamics, Phys. Rev. Lett. 3 (1959) 351.

[33] B. Zumino, Gauge properties of propagators in Quantum Electrodynamics, J. Math. Phys. 1 (1960) 1. 